\title{
Diagonal Function of k-Lucas Polynomials
}

\author{
Yogesh Kumar Gupta ${ }^{1, *}$, V. H. Badshah ${ }^{1}$, Mamta Singh $^{2}$, Kiran Sisodiya $^{1}$ \\ ${ }^{1}$ School of Studies in Mathematics, Vikram University Ujjain, (M. P.) India \\ ${ }^{2}$ Department of Mathematical Science and Computer applications, Bundelkhand University, Jhansi (U. P.) India \\ *Corresponding author: yogeshgupta.880@rediffmail.com
}

Received January 29, 2015; Revised March 10, 2015; Accepted April 19, 2015

\begin{abstract}
The Lucas polynomials are famous for possessing wonderful and amazing properties and identities. In this paper, Diagonal function of k-Lucas Polynomials is introduced and defined by $\mathrm{G}_{\mathrm{n}+1}(x)=\mathrm{kxG}_{\mathrm{n}}(x)+\mathrm{G}_{\mathrm{n}-2}(\mathrm{x}), \mathrm{n} \geq 1$. with $\mathrm{G}_{0}(x)=2$. and $\mathrm{G}_{1}(x)=1$. Some Lucas Polynomials, rising \& descending diagonal function and generating matrix established and derived by standard methods.
\end{abstract}

Keywords: Lucas Polynomials, rising diagonal function, descending diagonal function and generating matrix

Cite This Article: Yogesh Kumar Gupta, V. H. Badshah, Mamta Singh, and Kiran Sisodiya, "Diagonal Function of k-Lucas Polynomials.” Turkish Journal of Analysis and Number Theory, vol. 3, no. 2 (2015): 49-52. doi: 10.12691/tjant-3-2-3.

\section{Introduction}

The sequence $1,1,2,3,5 \ldots$, got its name as name as Fibonacci sequence by the Famous Mathematics Francois Edouard Lucas in 1876 [7].

Lucas also discovered a new Fibonacci like sequence with different initial condition call it, Lucas Sequence

$$
\mathrm{L}_{\mathrm{n}}=\mathrm{L}_{\mathrm{n}-1}+\mathrm{L}_{\mathrm{n}-2, \mathrm{n} \geq 2}
$$

with initial condition $\mathrm{L}_{0}=2, \mathrm{~L}_{1}=1$.

In 1965 Hoggatt, V.E. [5] has defined Lucas polynomials by recurrence relation. $\mathrm{L}_{\mathrm{n}+1}(x)=\mathrm{xL}_{\mathrm{n}}(x)+\mathrm{L}_{\mathrm{n}-1},(\mathrm{x})$, where

$$
\mathrm{L}_{0}(x)=2, \mathrm{~L}_{1}(x)=\mathrm{x},
$$

The first few Lucas polynomials are

$$
\begin{aligned}
& \mathrm{L}_{1}(x)=1 \cdot x^{1} \\
& \mathrm{~L}_{2}(x)=1 \cdot x^{2}+2 \cdot x^{0} \\
& \mathrm{~L}_{3}(x)=1 \cdot x^{3}+3 \cdot x^{1} \\
& \mathrm{~L}_{4}(x)=1 \cdot x^{4}+4 \cdot x^{2}+2 \cdot x^{0} \\
& \mathrm{~L}_{5}(x)=1 \cdot x^{5}+5 \cdot x^{2}+5 \cdot x^{1} \\
& \mathrm{~L}_{6}(x)=1 \cdot x^{6}+6 \cdot x^{4}+9 \cdot x^{2}+2 \cdot x^{0}, \\
& \mathrm{~L}_{7}(x)=1 \cdot x^{7}+7 \cdot x^{5}+4 \cdot x^{5}+7 \cdot x^{1}, \ldots
\end{aligned}
$$

In this paper, we are using the pair of sequence $\left\{G_{n}\right\}$ and $\left\{\mathrm{P}_{\mathrm{n}}\right\}$ for which,

$$
\begin{aligned}
\mathrm{G}_{\mathrm{n}+1}(x) & =\mathrm{kxG}_{\mathrm{n}}(x)+\mathrm{G}_{\mathrm{n}-1,}(\mathrm{x}), \mathrm{n} \geq 1 . \\
\mathrm{G}_{0} & =2, \mathrm{G}_{1}=1 \quad(\mathrm{x} \neq 0)
\end{aligned}
$$

$$
\begin{gathered}
\mathrm{P}_{\mathrm{n}+1}(x)=\mathrm{kxP}_{\mathrm{n}}(x)+\mathrm{P}_{\mathrm{n}-1,}(\mathrm{x}), \mathrm{n} \geq 1 \\
P_{0}=k, P_{1}=k x . \quad(\mathrm{x} \neq 0)
\end{gathered}
$$

where $\mathrm{k}$ is any positive integer. $\mathrm{k}=0,1,2,3$...

Using the equation (1.1) and (1.2) and made a rising diagonal function and descending Diagonal Functions.

\section{Sequence $\left\{G_{n}\right\}$ and $\left\{P_{n}\right\}$}

We have the pair of sequence $\left\{G_{n}\right\}$ and $\left\{P_{n}\right\}$ for which,

$$
\begin{array}{cc}
\mathrm{G}_{\mathrm{n}+1}(x)=\mathrm{kxG}_{\mathrm{n}}(x)+\mathrm{G}_{\mathrm{n}-1},(\mathrm{x}), \mathrm{n} \geq 1 . \\
\mathrm{G}_{0}=2, \mathrm{G}_{1}=1 \quad(\mathrm{x} \neq 0), \\
\mathrm{P}_{\mathrm{n}+1}(x)=\mathrm{kxP}_{\mathrm{n}}(x)+\mathrm{P}_{\mathrm{n}-1,}(\mathrm{x}), \mathrm{n} \geq 1 . \\
\mathrm{P}_{0}=\mathrm{k}, \mathrm{P}_{1}=\mathrm{kx} . \quad(\mathrm{x} \neq 0)
\end{array}
$$

The first few terms of the sequence $\left\{G_{n}\right\}$ are 2

$$
1
$$

$k x+2$

$k^{2} x^{2}+2 k x+1$

$$
\mathrm{k}^{3} x^{3}+2 k^{2} x^{2}+2 k x+2
$$$$
\mathrm{k}^{4} x^{4}+2 k^{3} x^{3}+3 k^{2} x^{2}+4 k x+1
$$$$
\mathrm{k}^{5} x^{5}+2 \mathrm{k}^{4} x^{4}+4 k^{3} x^{3}+6 k^{2} x^{2}+3 k x+2
$$

$$
\mathrm{k}^{6} x^{6}+2 \mathrm{k}^{5} x^{5}+5 \mathrm{k}^{4} x^{4}+8 k^{3} x^{3}+6 k^{2} x^{2}+6 k x+1
$$$$
\mathrm{k}^{7} x^{7}+2 \mathrm{k}^{6} x^{6}+6 \mathrm{k}^{5} x^{5}+10 \mathrm{k}^{4} x^{4}
$$$$
+10 k^{3} x^{3}+12 k^{2} x^{2}+4 k x+2
$$$$
\mathrm{k}^{8} x^{8}+2 \mathrm{k}^{7} x^{7}+7 \mathrm{k}^{6} x^{6}+12 \mathrm{k}^{5} x^{5}+15 \mathrm{k}^{4} x^{4}
$$

$$
+20 k^{3} x^{3}+10 k^{2} x^{2}+8 k x+1 .
$$

The first few terms of the sequence $\left\{P_{n}\right\}$ are 


$$
\begin{aligned}
& k \\
& k x \\
& k^{2} x^{2}+k \\
& \mathrm{k}^{3} x^{3}+k^{2} x+k x \\
& \mathrm{k}^{4} x^{4}+k^{3} x^{2}+2 k^{2} x^{2}+k \\
& \mathrm{k}^{5} x^{5}+\mathrm{k}^{4} x^{3}+3 k^{3} x^{3}+2 \mathrm{k}^{2} x+k x, \\
& \mathrm{k}^{6} x^{6}+\mathrm{k}^{5} x^{4}+4 \mathrm{k}^{4} x^{4}+3 \mathrm{k}^{3} x^{2}+3 \mathrm{k}^{2} x^{2}+k \\
& \mathrm{k}^{7} x^{7}+\mathrm{k}^{6} x^{5}+5 \mathrm{k}^{5} x^{5}+4 \mathrm{k}^{4} x^{3} \\
& \quad+6 k^{3} x^{3}+3 k^{2} x+k x \\
& \mathrm{k}^{8} x^{8}+\mathrm{k}^{7} x^{6}+6 \mathrm{k}^{6} x^{6}+5 \mathrm{k}^{5} x^{4} \\
& \quad+10 \mathrm{k}^{4} x^{4}+6 k^{3} x^{2}+4 k^{2} x^{2}+k .
\end{aligned}
$$

\section{Rising Diagonal Function}

Consider the rising diagonal function of $\mathrm{x}, \mathrm{U}_{\mathrm{n}}(\mathrm{x}), \mathrm{u}_{\mathrm{n}}(\mathrm{x})$ for (2.1) and (2.2) respectively,

$$
\begin{aligned}
\mathrm{U}_{1}(\mathrm{x}) & =1 \\
\mathrm{U}_{2}(\mathrm{x}) & =k x \\
\mathrm{U}_{3}(\mathrm{x}) & =k^{2} x^{2}+2 \\
\mathrm{U}_{4}(\mathrm{x}) & =k^{3} x^{3}+2 k x \\
\mathrm{U}_{5}(\mathrm{x}) & =k^{4} x^{4}+2 k^{2} x^{2}+1 \\
\mathrm{U}_{6}(\mathrm{x}) & =k^{5} x^{5}+2 k^{3} x^{3}+2 k x \\
\mathrm{U}_{7}(\mathrm{x}) & =k^{6} x^{6}+2 k^{4} x^{4}+3 k^{2} x^{2}+2 \\
\mathrm{U}_{8}(\mathrm{x}) & =k^{7} x^{7}+2 k^{5} x^{5}+4 k^{3} x^{3}+4 k x \\
\mathrm{U}_{9}(\mathrm{x}) & =k^{8} x^{8}+2 k^{6} x^{6}+5 k^{4} x^{4}+6 k^{2} x^{2}+1 . \\
\mathrm{u}_{1}(\mathrm{x}) & =k \\
\mathrm{u}_{2}(\mathrm{x}) & =k x^{2} \\
\mathrm{u}_{3}(\mathrm{x}) & =k^{2} x^{2} \\
\mathrm{u}_{4}(\mathrm{x}) & =k^{3} x^{3}+k \\
\mathrm{u}_{5}(\mathrm{x}) & =k^{4} x^{4}+k^{2} x \\
\mathrm{u}_{6}(\mathrm{x}) & =k^{5} x^{5}+k^{3} x^{3}+k x \\
\mathrm{u}_{7}(\mathrm{x}) & =k^{6} x^{6}+k^{4} x^{3}+2 k^{2} x^{2} \\
\mathrm{u}_{8}(\mathrm{x}) & =k^{7} x^{7}+k^{5} x^{4}+3 k^{3} x^{3}+k \\
\mathrm{u}_{9}(\mathrm{x}) & =k^{8} x^{8}+k^{6} x^{5}+4 k^{4} x^{4}+2 k^{2} x^{2}
\end{aligned}
$$

Now, we define

$$
\mathrm{U}_{0}(\mathrm{x})=u_{0}(x)=0 .
$$

from equation (3.1), (3.2) and (3.3) we get the following theorem:

Theorem (1). If $U_{n}(x)$ and $u_{n}(x)$ are rising diagonal functions of $x$ for sequence $\left\{G_{n}\right\}$ and $\left\{P_{n}\right\}$ respectively, than for, $n \geq 4$

$$
\mathrm{U}_{\mathrm{n}}(\mathrm{x})=k x U_{n-1}(x)+U_{n-4}(x) .
$$

\section{Special Case-I}

If $\mathrm{U}_{\mathrm{n}}(\mathrm{x})$ and $\mathrm{u}_{\mathrm{n}}(\mathrm{x})$ are rising diagonal functions of $\mathrm{x} f$ sequence $\left\{G_{n}\right\}$ and $\left\{P_{n}\right\}$ respectively, than for $n=3, n=4$.

$$
\mathrm{u}_{\mathrm{n}}(\mathrm{x})=k x u_{n-1}(x)+u_{n-3}(x) .
$$

\section{Descending Diagonal Function}

From (2.1) and (2.2), the descending diagonal function of $x, Q_{i}(x), q_{i}(x)$ are

$$
\begin{gathered}
\mathrm{Q}_{1}(\mathrm{x})=1 \\
\mathrm{Q}_{2}(\mathrm{x})=k x+1 \\
\mathrm{Q}_{3}(\mathrm{x})=(k x+1)^{2} \\
\mathrm{Q}_{4}(\mathrm{x})=(k x+1)^{3} \\
\mathrm{Q}_{5}(\mathrm{x})=(k x+1)^{4} \\
\mathrm{Q}_{6}(\mathrm{x})=(k x+1)^{5} \\
\mathrm{Q}_{7}(\mathrm{x})=(k x+1)^{6} \\
\mathrm{Q}_{8}(\mathrm{x})=(k x+1)^{7} \\
\mathrm{q}_{1}(\mathrm{x})=k \\
\mathrm{q}_{2}(\mathrm{x})=k x+k \\
\mathrm{q}_{3}(\mathrm{x})=(k x+k)(k x+1) \\
\mathrm{q}_{4}(\mathrm{x})=(k x+k)(k x+1)^{2} \\
\mathrm{q}_{5}(\mathrm{x})=(k x+k)(k x+1)^{3} \\
\mathrm{q}_{6}(\mathrm{x})=(k x+k)(k x+1)^{4} \\
\mathrm{q}_{7}(\mathrm{x})=(k x+k)(k x+1)^{5} \\
\mathrm{q}_{8}(\mathrm{x})=(k x+k)(k x+1)^{6}
\end{gathered}
$$

Now, we define

$$
\mathrm{Q}_{0}(\mathrm{x})=q_{0}(x)=0
$$

from (4.1), (4.2) and (4.3) we get for $n \geq 2$.

$$
\begin{aligned}
\mathrm{Q}_{\mathrm{n}}(\mathrm{x})=(k x+1) Q_{n-1} & =(k x+1)^{n-1} . \\
\mathrm{q}_{\mathrm{n}}(\mathrm{x}) & =(k x+1) q_{n-1} .
\end{aligned}
$$

from (4.4) and (4.5) we get the following theorem:

Theorem (2). If $\mathrm{Q}_{\mathrm{n}}(\mathrm{x})$ and $\mathrm{q}_{\mathrm{n}}(\mathrm{x})$ are descending diagonal function of $x$ for Sequence $\left\{G_{n}\right\}$ and $\left\{P_{n}\right\}$ respectively, than for $n>2$.
a) $\frac{\mathrm{Q}_{\mathrm{n}}}{\mathrm{Q}_{\mathrm{n}-1}}=\frac{q_{n}}{q_{n-1}}=(k x+1)$.
b) $\frac{\mathrm{Q}_{\mathrm{n}}}{\mathrm{q}_{\mathrm{n}}}=\frac{(k x+1)}{(k x+k)}$.

\section{Generating Matrix}

For the sequence $\left\{G_{n}\right\}$ defend in equation (1.1) we consider the matrix

Proof can be obtained by PMI's method so it is obvious. 


$$
A=\left[\begin{array}{cc}
k x & 1 \\
1 & 0
\end{array}\right]
$$

Since, the elements of this matrix are the member of the sequence of Fibonacci Polynomials. We call this matrix as Fibonacci matrix.

Theorem (3). For sequence $\left\{G_{n}\right\}$ we define $\mathrm{n} \geq 1, \mathrm{p} \geq 0$.

$$
\begin{aligned}
& \mathrm{G}_{\mathrm{n}+p}(x)=\mathrm{G}_{\mathrm{n}+1}(x) \mathrm{G}_{\mathrm{p}}(\mathrm{x})+G_{n}(x) G_{p-1}(x) \\
& \mathrm{G}_{\mathrm{n}+p}(x)=\mathrm{G}_{\mathrm{n}}(x) \mathrm{G}_{\mathrm{p}+1}(\mathrm{x})+G_{n-1}(x) G_{p}(x) .
\end{aligned}
$$

Proof. For sequence $\left\{G_{n}\right\}$, we have

$$
A=\left[\begin{array}{cc}
k x & 1 \\
1 & 0
\end{array}\right]
$$

Since, determinant of matrix A is -1 , therefore,

$$
\begin{gathered}
\operatorname{det} \mathrm{A}^{\mathrm{n}}=(\operatorname{det} A)^{n} \\
\operatorname{det} \mathrm{A}^{\mathrm{n}}=(-1)^{n} \\
A^{n}=\left[\begin{array}{cc}
G_{n+1}(x) & G_{n}(x) \\
G_{n}(x) & G_{n-1}(x)
\end{array}\right]
\end{gathered}
$$

Form equation (3.5.2) and (3.5.3), we get

$$
\mathrm{G}_{\mathrm{n}+1}(x) G_{n-1}(x)-G_{n}^{2}(x)=(-1)^{n}
$$

Since $A^{n+p}=A^{n} A^{p}$

$$
\begin{aligned}
& {\left[\begin{array}{cc}
G_{n+p+1}(x) & G_{n+p}(x) \\
G_{n+p}(x) & G_{n+p-1}(x)
\end{array}\right]} \\
& =\left[\begin{array}{cc}
G_{n+1}(x) & G_{n}(x) \\
G_{n}(x) & G_{n-1}(x)
\end{array}\right]\left[\begin{array}{cc}
G_{p+1}(x) & G_{p}(x) \\
G_{p}(x) & G_{p-1}(x)
\end{array}\right]
\end{aligned}
$$

After multiplying the matrices and equating the corresponding elements, we get

$$
\begin{aligned}
& \mathrm{G}_{\mathrm{n}+p}(x)=\mathrm{G}_{\mathrm{n}+1}(x) \mathrm{G}_{\mathrm{p}}(\mathrm{x})+G_{n}(x) G_{p-1}(x) \\
& \mathrm{G}_{\mathrm{n}+p}(x)=\mathrm{G}_{\mathrm{n}}(x) \mathrm{G}_{\mathrm{p}+1}(\mathrm{x})+G_{n-1}(x) G_{p}(x) .
\end{aligned}
$$

Theorem (4). For sequence $\left\{G_{n}\right\}$ we define $n \geq 1, p \geq 0$

$$
\begin{aligned}
& \mathrm{G}_{\mathrm{n}}(x)=\mathrm{G}_{\mathrm{n}+p+1}(x) \mathrm{G}_{-\mathrm{n}}(\mathrm{x})+G_{n+p}(x) G_{-(p+1)}(x) \\
& \mathrm{G}_{\mathrm{n}}(x)=\mathrm{G}_{\mathrm{n}+\mathrm{p}}(x) \mathrm{G}_{-\mathrm{p}+1}(\mathrm{x})+G_{n+p-1}(x) G_{-p}(x) .
\end{aligned}
$$

Proof. For sequence $\left\{G_{n}\right\}$, we have

$$
A=\left[\begin{array}{cc}
k x & 1 \\
1 & 0
\end{array}\right]
$$

If $A$ is any square Matrix, then we know that

$$
\mathrm{AA}^{-1}=\mathrm{I}
$$

Where I is identity matrix from equation (5.4) we get

$$
A^{-1}=\left[\begin{array}{cc}
0 & 1 \\
0 & -k x
\end{array}\right]
$$

$$
A^{-1}=\left[\begin{array}{cc}
G_{0}(x) & G_{-1}(x) \\
G_{-1}(x) & G_{-2}(x)
\end{array}\right] .
$$

By Mathematical induction, we have

$$
A^{-p}=\left[\begin{array}{cc}
G_{-(p-1)}(x) & G_{-p}(x) \\
G_{-p}(x) & G_{-(p+1)}(x)
\end{array}\right]
$$

Since $\mathrm{A}^{\mathrm{n}}=\mathrm{A}^{\mathrm{n}+\mathrm{p}} A^{-p}$

$$
\begin{aligned}
& {\left[\begin{array}{cc}
G_{n+p}(x) & G_{n}(x) \\
G_{n}(x) & G_{n-1}(x)
\end{array}\right]} \\
& =\left[\begin{array}{cc}
G_{n+p+1}(x) & G_{n+p}(x) \\
G_{n+p}(x) & G_{n+p-1}(x)
\end{array}\right]\left[\begin{array}{cc}
G_{-(p-1)}(x) & G_{-p}(x) \\
G_{-p}(x) & G_{-(p+1)}(x)
\end{array}\right]
\end{aligned}
$$

After multiplying the matrices and equating the corresponding elements, we get

$$
\begin{aligned}
& \mathrm{G}_{\mathrm{n}}(x)=\mathrm{G}_{\mathrm{n}+p+1}(x) \mathrm{G}_{-\mathrm{n}}(\mathrm{x})+G_{n+p}(x) G_{-(p+1)}(x) \\
& \mathrm{G}_{\mathrm{n}}(x)=\mathrm{G}_{\mathrm{n}+\mathrm{p}}(x) \mathrm{G}_{-\mathrm{p}+1}(\mathrm{x})+G_{n+p-1}(x) G_{-p}(x) .
\end{aligned}
$$

\section{Generating Matrix}

For the sequence $\left\{P_{n}\right\}$ defend in equation (1.1) we consider the matrix

$$
A=\left[\begin{array}{cc}
k x & 1 \\
1 & 0
\end{array}\right]
$$

since, the elements of this matrix are the members of the sequence of Fibonacci polynomials. We call this matrix as Fibonacci Matrix.

Theorem (5). For sequence $\left\{P_{n}\right\}$ we define $n \geq 1, r \geq 0$

$$
\begin{aligned}
& \mathrm{P}_{\mathrm{n}+r}(x)=\mathrm{P}_{\mathrm{n}+1}(x) \mathrm{P}_{\mathrm{r}}(\mathrm{x})+P_{r}(x) P_{r-1}(x) \\
& \mathrm{P}_{\mathrm{n}+r}(x)=\mathrm{P}_{\mathrm{n}}(x) \mathrm{P}_{r+1}(\mathrm{x})+P_{n-1}(x) P_{r}(x) .
\end{aligned}
$$

Proof. For sequence $\left\{\mathrm{P}_{\mathrm{n}}\right\}$, we have

$$
A=\left[\begin{array}{cc}
k x & 1 \\
1 & 0
\end{array}\right]
$$

Since, determinant of matrix $\mathrm{A}$ is -1 , there for,

$$
\begin{aligned}
\operatorname{det} \mathrm{A}^{\mathrm{n}} & =(\operatorname{det} A)^{n} \\
\operatorname{det} \mathrm{A}^{\mathrm{n}} & =(-1)^{n} .
\end{aligned}
$$

By mathematical induction

$$
A^{n}=\left[\begin{array}{cc}
P_{n+1}(x) & P_{n}(x) \\
P_{n}(x) & P_{n-1}(x)
\end{array}\right] .
$$

Form equation (3.6.2) and (3.6.3), we get

$$
\mathrm{P}_{\mathrm{n}+1}(x) P_{n-1}(x)-P_{n}^{2}(x)=(-1)^{n}
$$

Since $A^{n+r}=A^{n} A^{r}$ 


$$
\begin{aligned}
& {\left[\begin{array}{cc}
p_{n+r+1}(x) & p_{n+r}(x) \\
p_{n+r}(x) & p_{n+r-1}(x)
\end{array}\right]} \\
& =\left[\begin{array}{cc}
p_{n+1}(x) & p_{n}(x) \\
p_{n}(x) & p_{n-1}(x)
\end{array}\right]\left[\begin{array}{cc}
p_{r+1}(x) & p_{r}(x) \\
p_{r}(x) & p_{r-1}(x)
\end{array}\right]
\end{aligned}
$$

After multiplying the matrices and equating the corresponding elements, we get

$$
\begin{aligned}
& \mathrm{P}_{\mathrm{n}+r}(x)=\mathrm{P}_{\mathrm{n}+1}(x) \mathrm{P}_{\mathrm{r}}(\mathrm{x})+P_{n}(x) P_{r-1}(x) . \\
& \mathrm{P}_{\mathrm{n}+r}(x)=\mathrm{P}_{\mathrm{n}}(x) \mathrm{P}_{\mathrm{r}+1}(\mathrm{x})+P_{n-1}(x) P_{r}(x) .
\end{aligned}
$$

Theorem (6). For sequence $\left\{P_{n}\right\}$ we define $n \geq 1, r \geq 0$.

$$
\begin{aligned}
& \mathrm{P}_{\mathrm{n}}(x)=\mathrm{P}_{\mathrm{n}+r+1}(x) \mathrm{P}_{-\mathrm{r}}(\mathrm{x})+P_{n+r}(x) P_{-(r+1)}(x) . \\
& \mathrm{P}_{\mathrm{n}}(x)=\mathrm{P}_{\mathrm{n}+\mathrm{r}}(x) \mathrm{P}_{-r+1}(\mathrm{x})+P_{n+r-1}(x) P_{-r}(x) .
\end{aligned}
$$

Proof. For sequence $\left\{G_{n}\right\}$, we have

$$
A=\left[\begin{array}{cc}
k x & 1 \\
1 & 0
\end{array}\right]
$$

If $\mathrm{A}$ is any square Matrix, then we know that

$$
\mathrm{AA}^{-1}=\mathrm{I}
$$

Where I is identity matrix from equation (5.4) we get

$$
\begin{aligned}
A^{-1} & =\left[\begin{array}{cc}
0 & 1 \\
1 & -k x
\end{array}\right] \\
A^{-1} & =\left[\begin{array}{ll}
P_{n}(x) & P_{-1}(x) \\
P_{-1}(x) & P_{-2}(x)
\end{array}\right]
\end{aligned}
$$

By mathematical indication, we have

$$
A^{-r}=\left[\begin{array}{cc}
P_{-(r-1)}(x) & P_{-r}(x) \\
P_{-r}(x) & P_{-(r+1)}(x)
\end{array}\right]
$$

Since $A^{n}=A^{n+r} A^{-r}$

$$
\begin{aligned}
& {\left[\begin{array}{cc}
p_{n+1}(x) & p_{n}(x) \\
p_{n}(x) & p_{n-1}(x)
\end{array}\right]} \\
& =\left[\begin{array}{cc}
p_{n+r+1}(x) & p_{n+r}(x) \\
p_{n+r}(x) & p_{n+r-1}(x)
\end{array}\right]\left[\begin{array}{cc}
p_{-(r-1)}(x) & p_{-r}(x) \\
p_{-r}(x) & p_{-(r+1)}(x)
\end{array}\right] .
\end{aligned}
$$

After multiplying the matrices and equating the corresponding elements, we get

$$
\begin{aligned}
& \mathrm{P}_{\mathrm{n}}(x)=\mathrm{P}_{\mathrm{n}+r+1}(x) \mathrm{P}_{-\mathrm{r}}(\mathrm{x})+P_{n+r}(x) P_{-(r+1)}(x) . \\
& \mathrm{P}_{\mathrm{n}}(x)=\mathrm{P}_{\mathrm{n}+\mathrm{r}}(x) \mathrm{P}_{-r+1}(\mathrm{x})+P_{n+r-1}(x) P_{-r}(x) .
\end{aligned}
$$

\section{Conclusions}

In this paper Diagonal function k-Lucas Polynomials. Some basic rising diagonal function and descending diagonal function and generating matrix derived by standard method.

\section{Acknowledgement}

We would like to thank the anonymous referees for numerous helpful suggestions.

\section{References}

[1] A.F Hordam, Diagonal Function, the Fibonacci Quarterly, Vol. 16, 19-36.

[2] Alexandra Lupas Guide of Fibonacci and Lucas polynomials, Octagon Mathematics Magazine Vo. 17, No.1 (1999), 2-12.

[3] B.S.Porov, A note on the sum of Fibonacci and Lucas polynomials, The Fill Quarterly 1970, 428-438.

[4] D.V. Jaiswal, "Some Metric Properties of a Generalized Fibonacci Sequence”, Labdev Journal of Science and technology, India vol.11-A, No.1, 1973, 1-3.

[5] Jr. V.E. Hoggatt, Fibonacci and Lucas Numbers, Houshton Mifflin Company, Borton, 1965.

[6] Koshy, T. Fibonacci and Lucas number with application, Wiley, 2001.

[7] M. Catalan, An Identity for Lucas Polynomials, Fibonacci Quarterly Vol 43, No.1 2005.

[8] Singh, B., S. Teeth, and Harne Diagonal Function of Fibonacci Polynomials, chh. J. Sci. Tech 2005, 97-102.

[9] Vajda, S. Fibonacci and Lucas numbers and the golden section, Ellis Horwood Limited, Chi Chester, England, 1989.

[10] Vorobyou, N.N., The Fibonacci number, D.C. health company, Boston, 1963. 